Article

\title{
Can Coronaviruses Steal Genes from the Host as Evidenced in Western European Hedgehogs by EriCoV Genetic Characterization?
}

\author{
Luca De Sabato ${ }^{1}$, Ilaria Di Bartolo ${ }^{1}$, Maria Alessandra De Marco ${ }^{2, *}$, Ana Moreno ${ }^{3, *}(\mathbb{D}$, \\ Davide Lelli $^{3}{ }^{(D)}$, Claudia Cotti ${ }^{4}$, Mauro Delogu ${ }^{4,+}$ and Gabriele Vaccari ${ }^{1,+}{ }^{(D)}$ \\ 1 Department of Food Safety, Nutrition and Veterinary Public Health, Istituto Superiore di Sanità, \\ 00161 Rome, Italy; luca.desabato@iss.it (L.D.S.); ilaria.dibartolo@iss.it (I.D.B.); gabriele.vaccari@iss.it (G.V.) \\ 2 ISPRA Institute for Environmental Protection and Research, 40064 Ozzano dell'Emilia, Italy \\ 3 Virology Unit, Istituto Zooprofilattico Sperimentale della Lombardia e dell'Emilia-Romagna, \\ 25124 Brescia, Italy; davide.lelli@izsler.it \\ 4 Department of Veterinary Medical Sciences, University of Bologna, 40064 Ozzano dell'Emilia, Italy; \\ claudia.cotti@unibo.it (C.C.); mauro.delogu@unibo.it (M.D.) \\ * Correspondence: mariaalessandra.demarco@isprambiente.it (M.A.D.M.); \\ anamaria.morenomartin@izsler.it (A.M.); Tel.: +39-051-6512205 (M.A.D.M.); +39-030-2290347 (A.M.); \\ Fax: +39-051-796628 (M.A.D.M.) \\ + These authors share senior authorship.
}

Academic Editors: Alice Fusaro and Isabella Monne

Received: 4 December 2020; Accepted: 18 December 2020; Published: 20 December 2020

\begin{abstract}
Due to their need for living cells, viruses have developed adaptive evolutionary strategies to survive and perpetuate in reservoir hosts that play a crucial role in the ecology of emerging pathogens. Pathogenic and potentially pandemic betacoronaviruses arose in humans in 2002 (SARS-CoV, disappeared in July 2003), 2012 (MERS-CoV, still circulating in Middle East areas), and 2019 (SARS-CoV-2, causing the current global pandemic). As universally recognized, bats host ancestors of the above-mentioned zoonotic viruses. However, hedgehogs have been recently identified in Europe and Asia as possible reservoirs of MERS-CoV-like strains classified as Erinaceus coronavirus (EriCoV). To elucidate the evolution and genetics of EriCoVs, NGS (next generation sequencing) and Sanger sequencing were used to examine fecal samples collected in Northern Italy in 2018/2019 from 12 hedgehogs previously found EriCoV-positive by RT-PCR. By sequence analysis, eight complete EriCoV genomes, obtained by NGS, showed a high phylogenetic correlation with EriCoV strains previously reported in Eurasia. Interestingly, eight viral strains presented an additional ORF encoding for the CD200 ortholog located between the genes encoding for the Spike and the ORF3a proteins. The CD200 ortholog sequences were closely similar to the host CD200 protein but varying among EriCoVs. The result, confirmed by Sanger sequencing, demonstrates for the first time that CoVs can acquire host genes potentially involved in the immune-modulatory cascade and possibly enabling the virus to escape the host defence.
\end{abstract}

Keywords: Erinaceus europaeus; hedgehogs; betacoronaviruses; Erinaceus coronavirus; EriCoV; CD200 glycoprotein; CD200 ortholog; Betacoronavirus infection; Eulipotyphla

\section{Introduction}

Coronaviruses (CoVs), classified as belonging to the Coronaviridae family, are a heterogeneous group of viruses with RNA (26-32 kb in length) single strand genomes which have been considered for a long time to be restricted to animals, in which that can cause respiratory, gastrointestinal, and other symptoms of variable severity [1]. In humans, $\mathrm{CoVs}$ are the second cause of respiratory disease causing 
about $15 \%$ of common colds worldwide [2]. Since 2002, three highly virulent strains classified into two subgenera (Sarbecovirus and Merbecovirus) of the Betacoronavirus (Beta-CoV) genus have caused severe respiratory diseases leading to thousands of infections and deaths worldwide. The Sarbecovirus subgenus includes the $\mathrm{CoV}$ species causing the severe acute respiratory syndrome (SARS-CoV) and the SARS-CoV-2, emerged in China in 2002 and in December 2019, respectively. The COVID-19 pandemic caused by SARS-CoV-2 is still ongoing. In 2012, the Middle East respiratory syndrome coronavirus (MERS-CoV), belonging to the Merbecovirus subgenus, was first reported in The Arabian Peninsula where it still circulates $[3,4]$.

The ability of CoVs to change fast by recombination, deletion or by acquiring mutations enables the virus to jump among different species and successfully adapts to new hosts [5]. Since the SARS epidemic, it has become evident that several bat species host possible ancestors of these three zoonotic betacoronaviruses, mainly found in the Rinolophidae (SARS-CoV and SARS-CoV-2 precursors) and Vespertilionidae (MERS-CoV precursors) families of the order Chiroptera [6-10].

Hedgehogs are mammals belonging to the Eulipotyphla order which is phylogenetically related to the Chiroptera order [11] and have been recently identified as possible reservoir of MERS-CoV-like strains in Europe and Asia [12-16]. In particular, within the genus Erinaceus two species have been suggested as reservoirs of novel $\mathrm{CoVs}$ which have been clustered into a separate Betacoronavirus clade, among Merbecovirus members. The Western European hedgehogs' CoVs were classified into the novel $\mathrm{CoV}$ species Erinaceus coronavirus (EriCoV) based on threshold value of $90 \%$ pairwise amino acid distance in the conserved domains of replicase polyprotein [17]. Up to date, the EriCoVs in hedgehogs have been detected in Germany, France, Great Britain, and Italy in Erinaceus europaeus [12-14] and a novel species named HKU31 has been reported in China in Erinaceus amurensis [15].

As previously stated, the ancient mammalian taxa of Chiroptera and Eulipotyphla show a close phylogenetic relationship $[18,19]$. Interestingly, common origin between bats and hedgehog merbecoviruses has also been hypothesized. In particular, the close similarity observed in the spike regions suggests that the bat viruses may have originated from a recombination event between $\mathrm{CoV}$ strains from the two animal hosts [15]. Bat and hedgehog species living in the temperate zone, such as the Italian ones [20-22], are mainly mammalian hibernators, sharing nocturnal habits and a mainly insectivorous diet. Furthermore, MERS-like CoVs have been previously detected in Italian bats [23].

After the first identification of EriCoV, several EriCoVs have been detected but only for five strains found in Germany, UK and China full genomes are available $[12,14,15]$. Thus, the availability of additional EriCoV full genome sequences could be important in elucidating the evolution and genetics of EriCoVs and the role of hedgehogs as reservoir and possible chronic shedding carrier of these potentially emerging RNA viruses.

For the above-mentioned reasons, the next generation sequencing approach was used to fully sequence genomes of betacoronaviruses detected in hedgehogs in Northern Italy in order to elucidate evolution, genetics of EriCoVs and to evaluate their role in public and veterinary health implications.

\section{Materials and Methods}

\subsection{Samples}

Between November 2018 and January 2019, 12 injured hedgehogs (Erinaceus europaeus) were found in both urban areas (cities, towns, villages, and suburbs) and rural areas in two provinces of the Emilia-Romagna region (Northern Italy). The hedgehogs were non-invasively sampled at a wildlife treatment and rehabilitation center and fecal samples were collected. Animals tested pan-CoV positive by RT-PCR [16] were enrolled in the study (Table 1). Total RNA was extracted by QIAmp Viral Mini kit (Qiagen, Milan, Italy) stored at $-80^{\circ} \mathrm{C}$ or immediately used. 
Table 1. Summary of NGS results and CD200 detection obtained by sequencing the 12 Erinaceus coronavirus (EriCoVs) from hedgehogs sampled between November 2018 and January 2019. Sites of collection are also reported.

\begin{tabular}{|c|c|c|c|c|c|c|}
\hline Sample ID & $\begin{array}{l}\text { Municipality } \\
\text { (Province) }^{1}\end{array}$ & $\begin{array}{l}\text { Recovery } \\
\text { Site }^{2}\end{array}$ & $\begin{array}{c}\text { Total NGS } \\
\text { Reads }\end{array}$ & $\begin{array}{c}\mathbf{N}^{\circ} \text { CoVs } \\
\text { Reads }\end{array}$ & $\begin{array}{c}\% \text { of } \\
\text { Genome } \\
\text { Built }\end{array}$ & $\begin{array}{c}\text { CD200 } \\
\text { Ortholog }\end{array}$ \\
\hline $116988 / 1$ & Budrio (BO) & R.A. & $1,521,953$ & 13,567 & $100 \%$ & - \\
\hline $50265 / 1$ & Bentivoglio (BO) & R.A. & $1,246,304$ & 13,094 & $100 \%$ & $\mathrm{Y}$ \\
\hline $50265 / 3$ & $\begin{array}{l}\text { Sala Bolognese } \\
\text { (BO) }\end{array}$ & U.A. & $1,531,553$ & 45 & - & - \\
\hline $50265 / 4$ & Bologna (BO) & U.A. & $1,679,321$ & 320 & - & $\mathrm{Y}$ \\
\hline $50265 / 10$ & Ferrara $(\mathrm{FE})$ & R.A. & 628,517 & 135 & - & $\mathrm{Y}$ \\
\hline $50265 / 11$ & Ferrara (FE) & R.A. & $1,506,517$ & 174,733 & $100 \%$ & $\mathrm{Y}$ \\
\hline $50265 / 12$ & Bentivoglio (BO) & U.A. & 979,563 & 78,706 & $100 \%$ & $\mathrm{Y}$ \\
\hline $50265 / 13$ & Granarolo (BO) & U.A. & $1,635,986$ & 16,679 & $100 \%$ & $\mathrm{Y}$ \\
\hline $50265 / 15$ & Imola (BO) & U.A. & $1,046,044$ & 85,354 & $100 \%$ & - \\
\hline $50265 / 16$ & Bentivoglio (BO) & U.A. & $1,406,884$ & 89 & - & - \\
\hline $50265 / 17$ & Bologna (BO) & U.A. & $1,677,168$ & 136,279 & $100 \%$ & $\mathrm{Y}$ \\
\hline $50265 / 19$ & Bologna (BO) & U.A. & $1,627,896$ & 753,529 & $100 \%$ & $\mathrm{Y}$ \\
\hline
\end{tabular}

${ }^{1}$ sites of animal findings; BO, Bologna; FE, Ferrara. ${ }^{2}$ R.A., rural area; U.A., urban area. ${ }^{3}$ Y present; - absent.

\subsection{Whole-Genome Sequencing}

Libraries were prepared as previously described by Moreno et al. [23] following the sequence independent single primer amplification (SISPA) [24]. The RNA was reverse transcribed by SuperScript IV Reverse Transcriptase (Thermo Fisher Scientific, Monza, Italy) following the manufacturer's instructions. The second strand of cDNA was synthesized by DNA Polymerase I Large (Klenow) Fragment (Promega, Milan, Italy) and the Klenow product amplified by the PCRBIO HiFi Polymerase (PCR BIOSYSTEM, Resnova s.r.l, Roma, Italy). The purified cDNA was digested with EcoRV enzyme (New England BioLabs, Pero, MI, Italy) and the libraries prepared by NEBNext Fast DNA Library Prep Set for Ion Torrent (New England BioLabs) size selecting the $400 \mathrm{nt}$ amplicons. The emulsion PCR and the sequencing run were performed using the Ion 520 \& Ion 530 Kit-OT2 according to the instructions (Ion Gene Studio S5 and Ion 530 Chip).

\subsection{Complete Genome Assembling}

Reads obtained by Ion Gene Studio S5 were analysed using the Galaxy Aries online tool (https://aries.iss.it/). The reads were checked, cleaned up and trimmed using the FastQC Read Quality reports and FASTQ positional and quality trimming. The de novo assembly was performed using the default parameters and excluding contigs shorter than 1000 bases by Spades 3.0. Reads were also mapped against the EriCoV reference genome (ErinaceusCoV/2012-174/GER/2012, NC_039207.1) using Bowtie2 software and the BAM output visualized by IGV software. The Open Reading Frames (ORFs) were predicted using the online tool ORF Finder (NCBI, http://www.ncbi.nlm.nih.gov/gorf/gorf.html). The complete genome Accession numbers on NCBI database were: ErinaceusCoV/Italy/116988-1/2018, MW246795; ErinaceusCoV/Italy/50265-19/2018， MW246796; ErinaceusCoV/Italy/50265-1/2018, MW246797; ErinaceusCoV/Italy/50265-17/2018, MW246798; ErinaceusCoV/Italy/50265-11/2019, MW246799; ErinaceusCoV/Italy/50265-12/2019, MW246800; ErinaceusCoV/Italy/50265-13/2019, MW246801; ErinaceusCoV/Italy/50265-15/2019, MW246802.

\subsection{Recombination Analysis}

Recombination analysis was performed with Simplot software version 3.5. by bootscan analysis. Complete genomes among Merbecovirus members were analysed with 1000 bootstrap replicates, a sliding window of 500 nucleotides and 200 nucleotides moving steps. 


\subsection{CD200 Detection by Reverse Transcription-PCR}

Based on the alignment of the complete genome sequences of Italian EriCoVs, two primer pairs were designed to target the CD200 ortholog. The primer pairs were designed on the $\mathrm{CoV}$ genomes in the $5^{\prime}$ and $3^{\prime}$ flanking region of the CD200 gene allowing to amplify the $3^{\prime}$ end of the spike gene, the CD200 and the $5^{\prime}$ of ORF3a gene. The primer pairs were named CD200Fw (5'-TGGATGTGGCACTAGTTGTC-3'; position 25,507 on NC_039207.1; ErinaceusCoV/2012-174/GER/2012) and CD200Rw (5'-CTGGATATTAGGAGCTGTGT-3'; position 26000). The Reverse Transcription-PCR (RT-PCR) amplification was performed using the QIAGEN One-Step RT-PCR Kit (Qiagen) following the manufacturer's instruction under the following amplification steps: $50{ }^{\circ} \mathrm{C}$ for $30^{\prime}, 95^{\circ} \mathrm{C}$ for $15^{\prime}$ and 40 cycles of $95^{\circ} \mathrm{C}$ for $30^{\prime \prime}, 58{ }^{\circ} \mathrm{C}$ for $30^{\prime \prime}$, $72{ }^{\circ} \mathrm{C}$ for $2^{\prime}$, and a final step of $72{ }^{\circ} \mathrm{C}$ for $7^{\prime}$. The amplicon obtained was $500 \mathrm{bp}$ fragment if the CD200 ortholog was not present in the EriCoV genome. If the CD200 ortholog was present in the genome, and based on the insertion site, the expected size of the fragment will be $>800 \mathrm{bp}$. The PCR products were analysed on 1\% agarose gel. The DNA amplicons were sequenced using the same PCR primers used for the one step RT-PCR by Eurofins Genomic (Milano, Italy). The CD200 ortholog sequences Accession numbers on NCBI database were: ErinaceusCoV/Italy/50265-13/2019, MW294203; ErinaceusCoV/Italy/50265-12/2019, MW294204; ErinaceusCoV/Italy/50265-17/2018, MW294205; ErinaceusCoV/Italy/50265-4/2018, MW294206; ErinaceusCoV/Italy/50265-10/2018, MW294207; ErinaceusCoV/Italy/50265-1/2018, MW294208.

\subsection{Phylogenetic Analysis}

Sequence alignments, nucleotide/amino acid pairwise identities, and phylogenetic analysis (maximum likelihood) were performed using the MEGA7 software. Phylogenetic analyses were performed using the general time reversible model with a Gamma distribution and Invariant sites $(\mathrm{GTR}+\mathrm{G}+\mathrm{I})$ with 1000 bootstrap replication as suggested by MEGA7 modeltest.

The most recent common ancestor (tMRCA) of Merbecovirus was estimated using the complete genome sequences and excluding the recombinant strains. The strict clock model with a constant-size coalescent tree prior was applied using the BEAST version 1.8.4 software (http://evolve. zoo.ox.ac.uk/beast/). The information about sampling dates were obtained from NCBI database (https://www.ncbi.nlm.nih.gov/nucleotide/) or published information. The generalized stepping-stone sampling (GSS) was used for model selection analysis and GTR + G + I as a substitution model. The Markov chain Monte Carlo (MCMC) was run with 100 million steps along and sampling every 10,000 steps. The convergence of MCMC chains was checked using Tracer v.1.7.1 checking ESS values $>200$ for each estimated parameter. The maximum clade credibility (MCC) tree was obtained from the tree posterior distribution using TreeAnnotator after 10\% burn-in and tree displayed by FigTree software (http://tree.bio.ed.ac.uk/software/figtree/).

\subsection{Structural Modelling}

Analysis of the spike and CD200 proteins and the prediction of the secondary structure were performed using SWISS-MODEL (https://swissmodel.expasy.org) based on default parameters. EriCoV-RBD models were built with the MERS-RBD/human CD26 complex (4KR0). The structure and interacting residues of RBD analysis was performed in the models and analyzed and highlighted using Chimera software [25] Secondary structure elements are defined based on an ESPript (http://espript.ibcp.fr) algorithm [26] and compared to mouse CD200 (PDB: 4bfi).

\section{Results}

\subsection{Complete Genome Sequences and Genome Organization}

To determine the evolutionary relationship with other merbecoviruses, 12 fecal samples collected from Western European hedgehogs (Erinaceus europaeus) previously tested positive for CoVs [16] were 
subject to NGS to obtain complete genome sequences. From eight out of 12 samples, the sequence reads obtained enabled to build complete genomes (Table 1).

Sequence analysis of the full genomes by BLASTn showed the highest similarity to betacoronaviruses to the Merbecovirus subgenus. The complete genomes obtained are approximately $30 \mathrm{~kb}$ long with $\mathrm{G}+\mathrm{C}$ content of approximately $37.7 \%$. The genome organization of Italian hedgehog strains is similar to other members of the Merbecovirus subgenus: the 5' UTR-ORF1ab-spike-ORF3a-ORF3b-ORF4a-ORF4b-ORF5-envelope (E)-membrane (M)-nucleocapsid (N)-ORF8b and the $3^{\prime}$ UTR. The ORF1ab gene has the predicted slippery sequence "UUUAAAC" involved in synthesis of the pp1ab polyprotein by ribosomal frameshift. A putative leader transcription regulatory sequence (TRS-L) 5'-AACGAAC- $3^{\prime}$ preceded ORF1ab and seven putative transcription regulatory sequences body (TRS-B) have been identified for each ORF except for ORF4b and ORF3b, representing signals for the discontinuous transcription of subgenomic mRNAs (sgmRNAs) (Supplementary Table S1). Within the pp1ab protein, the size and genomic localization of the non-structural protein (NSP 1-16) were predicted with the expected cleavage sites of the "main protease" 3C-like protease (3CLpro) and the papain-like protease (Supplementary Table S2).

The Italian strains were collected from animals found in seven municipalities and genomes with almost identical nucleotide sequences from $95 \%$ to $97 \%$ identities (nt. id.) were obtained. Both identical $(100 \%)$ and divergent $(50265 / 1$ and 50265/12; 96.1\%) viral sequences were detected in each municipality. In four of them, only one strain was detected. Differently the same strain was detected (50265/1 and $50265 / 13100 \%$ nt. id.) in animals captured in two different municipalities.

By sequence comparison, the viral sequences closest to the Italian sequence strains were from Western European hedgehog strains detected in German and Great Britain (MK6796601) displaying 92\% and 91\% nt. id. (NC_039207.1; ErinaceusCoV/2012-174/GER/2012), respectively, followed by 81\% nt. id. with Erinaceus amurensis hedgehog coronavirus HKU31 (Ea-HedCoV HKU31) strain_F6 described in China (MK907286.1). The reference strains of MERS-CoVs (NC_019843.3, MERS_2c_EMC2012;) and the bat CoVs HKU4-1 and HKU5-1 (EF065505.1; EF065509.1) were more distant displaying $70 \%$ nt. id., $68 \%$ nt. id. and $67 \%$ nt. id., respectively. Similar results were obtained calculating the amino acids identities between the predicted protein of Italian strains and betacoronaviruses (Supplementary Table S3). The aa. id. values calculated comparing domains in the ORF1ab of the Italian strains with the hedgehog HKU31, MERS-CoV and MERS-CoV related sequence strains were lower than the threshold established for CoVs classification (90\%), suggesting the classification in the EriCoV species represented by the prototype strain ErinaceusCoV/2012-174/GER/2012.

\subsection{CD200 Ortholog Detection by RT-PCR}

By reconstruction and analyses of the full genome sequences in six out of eight Italian strains $(50265 / 1,50265 / 11,50265 / 12,50265 / 13,50265 / 17,50265 / 19)$ an additional ORF was retrieved, placed between genes encoding for the Spike and the ORF3a protein preceded by TRS-B. The length of the novel ORF varied between 411 and $630 \mathrm{bp}$ depending on the strain, encoding for a predicted peptide of 137-210 aa. By comparing the 6 sequences against the NCBI database the highest nucleotide identity ranged from $87.6 \%$ to $89.5 \%$ to the host Erinaceus europaeus CD200 gene and the highest aa. id. was of $74.1 \%$ to $80.3 \%$ to the translated host CD200 predicted protein (also named OX-2 membrane glycoprotein) (XP_007516410.2) (Supplementary Table S4). This result suggested the identification of the CD200 ortholog sequence in the genomes of the 6 fully sequenced EriCoVs.

To evaluate the presence of CD200 in the other 4 EriCoV strains for which the full genomes were not obtained, ad hoc primer pairs were designed onto the EriCoVs genome regions flanking the CD200 ortholog. The RT-PCR was designed to amplify a fragment $>800 \mathrm{bp}$ if the CD200 gene was inserted in the genome and a 500bp if it was not. By RT-PCR, two strains showed the amplification of $1200 \mathrm{bp}$ fragment that as confirmed by sequencing using the Sanger method corresponds to the CD200 ortholog sequence gene and mapped in the same position of the genome (between the Spike and ORF3a genes) 
(Table 1). Differently, four strains did not include the CD200 ortholog, as confirmed by sequencing the $500 \mathrm{bp}$ fragments obtained by RT-PCR (Table 1).

To confirm the presence of the CD200 on six strains sequenced by NGS, the same RT-PCR followed by Sanger sequencing was also performed on. The sequences obtained by both sequence approaches were identical (100\% nt. id.) each other and the site of insertion of the CD200 between the genes encoding for the Spike and the ORF3a protein was also confirmed. The 8 CD200 ortholog sequences displayed from 78.2 to $100 \%$ nt. id. each other and $67.9 \%$ to $100 \%$ aa. id. (Supplementary Table S4). Only two sequences were identical. Furthermore, by amino acid alignment in one strain (50265/12) a deletion of 189 nucleotides in the CD200 was present. By clustering in the Maximum Likelihood phylogenetic tree built including the CD200 sequences reported in mammals and several viral orthologs, the evolutionary correlation between EriCoV CD200 orthologs and the Erinaceus europaeus CD200 was confirmed (Supplementary Figure S1).

Since no crystal of the CD200 protein from Erinaceus species is available, the predicted secondary structure of the mouse CD200 was used for the analyses. Comparison of the structures revealed that CD200 orthologs found in Italian EriCoVs showed a similar structure to the mouse CD200 including 9 beta strands (named A,B,C, $C^{\prime}, C^{\prime \prime}, \mathrm{D}, \mathrm{E}, \mathrm{F}, \mathrm{G}$ ) in the first Ig-like domain of CD200, which is the domain involved in the binding of the host receptor CD200R. The 50265/12 strain predicted CD200 structure lacked the 8th and 9th (F, G) beta strands (Supplementary Figure S2). In addition, the amino acids stretch involved in the binding interface of complex CD200/CD200R are highly conserved between mouse CD200, hedgehogs and the CD200 orthologs of EriCoV (Table 2).

Table 2. Multiple sequence alignment showing variations in the key binding amino acid residues in CD200/CD200R binding site. Conserved residues are highlighted in red.

\begin{tabular}{llllllllllllllll}
\hline CD200 & \multicolumn{10}{c}{10} \\
\hline Mouse & T33 & Q35 & N44 & T47 & N94 & S41 & P42 & Q57 & T95 & G97 & S98 & Q99 & K100 & F96 \\
\hline 50265/1 & V52 & Q54 & N63 & T66 & N113 & G60 & P61 & Q76 & T114 & G116 & S117 & G118 & K119 & F115 \\
50265/4 & T52 & Q54 & N63 & T66 & N112 & I60 & P61 & Q76 & T113 & - & S115 & I116 & K120 & Y114 \\
50265/10 & T52 & Q54 & N63 & T66 & N113 & S60 & T61 & Q76 & T114 & G116 & S117 & G118 & S119 & F115 \\
50265/11 & T52 & Q54 & N63 & T66 & N113 & G60 & P61 & Q76 & I114 & G116 & P117 & V118 & M119 & F115 \\
50265/12 & T52 & T54 & N63 & T66 & -1 & I60 & P61 & L76 & - & - & - & - & - & - \\
50265/14 & V52 & Q54 & N63 & T66 & N113 & G60 & P61 & Q76 & T114 & G116 & S117 & G118 & K119 & F115 \\
50265/17 & T52 & Q54 & N63 & T66 & N113 & V60 & P61 & Q76 & T114 & G116 & S117 & G118 & R119 & F115 \\
50265/19 & T52 & Q54 & N63 & T66 & N113 & S60 & P61 & Q76 & T114 & - & S116 & R117 & K119 & F115 \\
Hedgehog T60 & Q62 & N71 & T74 & N121 & S68 & P69 & Q84 & T122 & G124 & S125 & G126 & K127 & F123 \\
\hline
\end{tabular}

\subsection{Phylogenetic Analysis of the Complete Genomes}

Recombination events were excluded between Italian EriCoVs and either Merbecovirus or Italian bat strains (MG596803; Bat-CoV/P.khulii/Italy/206645-63/2011). The phylogenetic tree built using the complete genomes (Figure 1) showed the clustering of the Italian $\mathrm{CoV}$ hedgehog strains with the Hedgehog coronavirus 1 strain (MK679660.1), the ErinaceusCoV/2012-174/GER/2012 (NC_039207.1) and ErinaceusCoV/2012-216/GER/2012 (KC545386.1) while HKU31 and the other bat MERS-CoV related strains were not included in the cluster of EriCoVs.

The time of the most recent common ancestor (tMRCA) of Merbecovirus, separation of MERS and MERS related species from the EriCoV cluster was approximately 1447 years ago with highest posterior density (HPD) regions at $95 \%$ of 717 to 2390 . The European EriCoVs originated approximately 190 years ago (95\% HPD: 94 to 313) and the MERS-CoV and MERS-CoV related strains were separated from bat CoVs HKU4 and HKU5 1229 years ago (95\% HPD: 610 to 2036) (Supplementary Figure S3). 


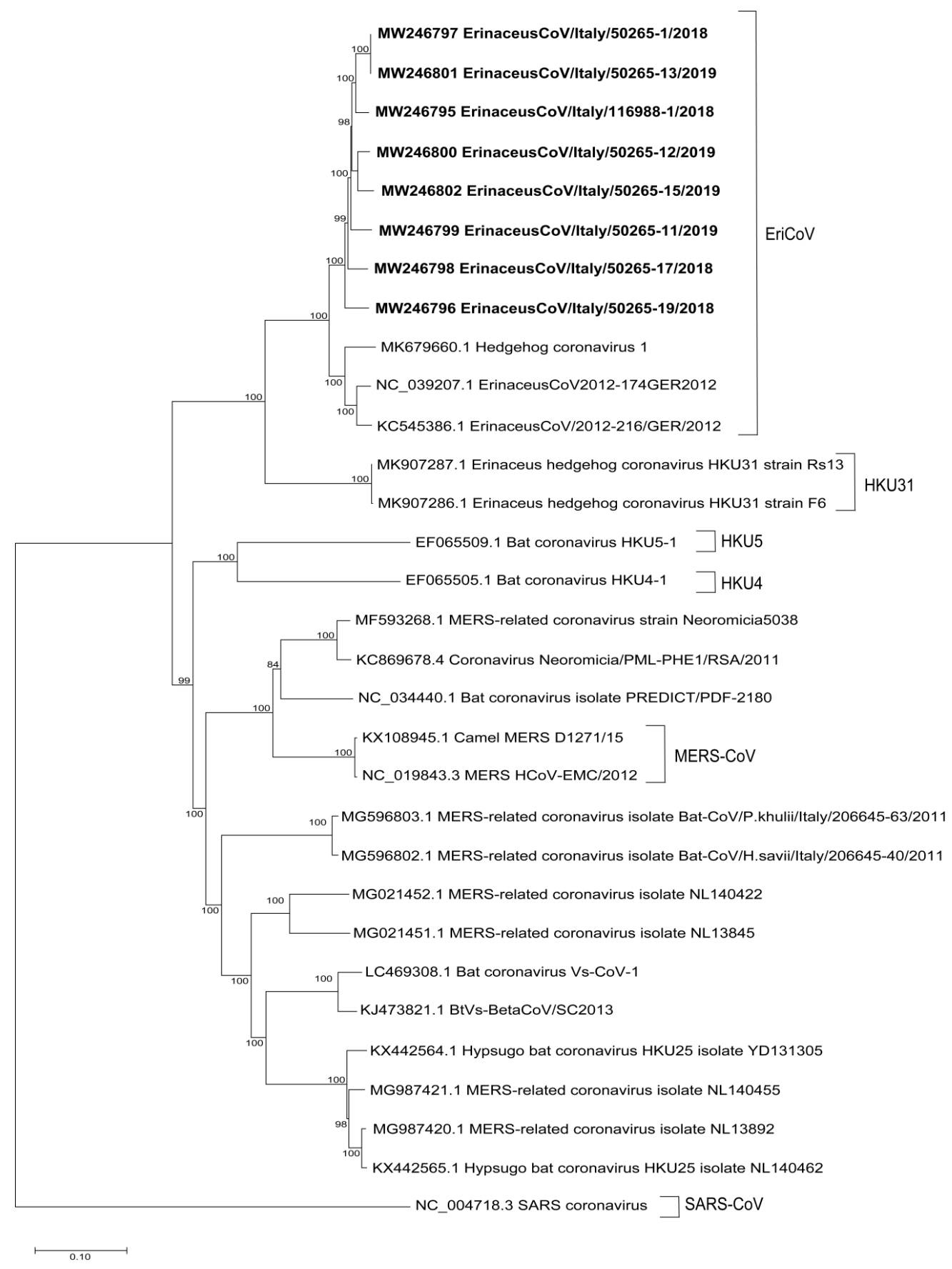

Figure 1. Maximum Likelihood phylogenetic tree of the Merbecovirus subgenus complete genomes including the eight Italian EriCoV strains. The tree was built using the GTR+G+I model and 1000 bootstrap replicates with supported nodes $(>70 \%)$. The entries are reported as: Accession number and the sequence name.

\subsection{Spike Protein Sequence Analysis}

Similar results to the analyses with the 6 whole genomes were observed for the spike protein which shared the highest identity $92 \%$ aa. id. with Erinaceus CoV/2012-174/GER/2012, 91\% aa. id. with Hedgehog coronavirus 1 strain and only $58 \%$ with MERS-CoVs. The same amino acid identity was obtained by analysing the RBD region.

The Italian EriCoVs RBD had four amino acids (Y491, P502, D525 and V540) out of 12 conserved residues needed to MERS-CoV for the binding to the human hDPP4 receptor (Table 3). However, 
comparing the structure of the EriCoVs with MERS-CoVs, the spike proteins lack the extended loop located between $\beta 6$ and $\beta 7$ needed for the interaction of hDPP4.

Table 3. Multiple sequence alignment showing variations in key amino acid binding residues of the Spike protein. Conserved residues are highlighted in red.

\begin{tabular}{|c|c|c|c|c|c|c|c|c|c|c|c|c|c|c|c|c|}
\hline MERS-CoV ${ }^{1}$ & $\begin{array}{c}Y \\
499\end{array}$ & $\begin{array}{c}N \\
501\end{array}$ & $\begin{array}{c}\mathrm{K} \\
502\end{array}$ & $\begin{array}{c}\text { L } \\
506\end{array}$ & $\begin{array}{c}\text { D } \\
510\end{array}$ & $\begin{array}{c}R \\
511\end{array}$ & $\begin{array}{c}E \\
513\end{array}$ & $\begin{array}{c}\text { P } \\
515\end{array}$ & $\begin{array}{r}W \\
535\end{array}$ & $\begin{array}{c}E \\
536\end{array}$ & $\begin{array}{c}\text { D } \\
537\end{array}$ & $\begin{array}{c}\text { D } \\
539\end{array}$ & $\begin{array}{c}Y \\
540\end{array}$ & $\begin{array}{c}R \\
542\end{array}$ & $\begin{array}{c}W \\
553\end{array}$ & $\begin{array}{c}\mathrm{V} \\
555\end{array}$ \\
\hline $\begin{array}{c}\text { Italian } \\
\text { EriCoVs }\end{array}$ & $\begin{array}{c}Y \\
491\end{array}$ & $\begin{array}{c}S \\
493\end{array}$ & $\begin{array}{c}\mathrm{R} \\
494\end{array}$ & - & $\begin{array}{c}G \\
500\end{array}$ & $\begin{array}{c}\mathrm{K} \\
501\end{array}$ & - & $\begin{array}{c}\mathrm{P} \\
502\end{array}$ & $\begin{array}{c}\mathrm{K} \\
523\end{array}$ & $\begin{array}{c}\mathrm{K} \\
524\end{array}$ & $\begin{array}{c}\mathrm{D} \\
525\end{array}$ & - & - & $\begin{array}{c}\mathrm{G} \\
530\end{array}$ & $\begin{array}{c}\mathrm{Y} \\
538\end{array}$ & $\begin{array}{c}\mathrm{V} \\
540\end{array}$ \\
\hline HKU $31^{2}$ & $\begin{array}{c}Y \\
491\end{array}$ & $\begin{array}{c}\mathrm{S} \\
493\end{array}$ & $\begin{array}{c}\mathrm{R} \\
494\end{array}$ & $\begin{array}{c}\mathrm{G} \\
497\end{array}$ & $\begin{array}{c}\mathrm{K} \\
501\end{array}$ & $\begin{array}{c}\mathrm{P} \\
502\end{array}$ & - & $\begin{array}{c}\mathrm{L} \\
504\end{array}$ & $\begin{array}{c}\mathrm{N} \\
524\end{array}$ & $\begin{array}{c}\mathrm{D} \\
525\end{array}$ & $\begin{array}{c}\mathrm{V} \\
526\end{array}$ & - & - & $\begin{array}{c}\mathrm{D} \\
527\end{array}$ & $\begin{array}{c}\mathrm{F} \\
538\end{array}$ & $\begin{array}{c}\text { I } \\
540\end{array}$ \\
\hline HKU4 ${ }^{3}$ & $\begin{array}{c}Y \\
503\end{array}$ & $\begin{array}{c}\mathrm{S} \\
505\end{array}$ & $\begin{array}{c}K \\
506\end{array}$ & $\begin{array}{c}\mathrm{L} \\
510\end{array}$ & $\begin{array}{c}\mathrm{N} \\
514\end{array}$ & $\begin{array}{c}\mathrm{Q} \\
515\end{array}$ & $\begin{array}{c}E \\
518\end{array}$ & $\begin{array}{c}\mathrm{P} \\
520\end{array}$ & $\begin{array}{c}\mathrm{S} \\
540\end{array}$ & $\begin{array}{c}\mathrm{E} \\
541\end{array}$ & $\begin{array}{c}\mathrm{D} \\
542\end{array}$ & $\begin{array}{c}\mathrm{Q} \\
544\end{array}$ & $\begin{array}{c}\mathrm{V} \\
545\end{array}$ & $\begin{array}{c}\mathrm{K} \\
547\end{array}$ & $\begin{array}{c}\mathrm{L} \\
558\end{array}$ & $\begin{array}{c}\mathrm{I} \\
560\end{array}$ \\
\hline
\end{tabular}

${ }^{1}$ Accession number: NC_019843.3, HCoV-EMC/2012. ${ }^{2}$ Accession number: MK907286.1, HKU31 strain F6. ${ }^{3}$ Accession number: EF065505.1, HKU4-1.

\section{Discussion}

Eight genomes of EriCoV strains were completely sequenced by NGS in order to evolutionarily characterize Merbecovirus members identified in Italian hedgehogs whose role as natural reservoirs and chronic carriers of betacoronavirus was previously hypothesized. Interestingly, genome analyses showed the common ORFs reported for other Merbecovirus strains and an additional ORF encoding the homologue of the CD200 of Erinaceus europaeus species, in six out of the eight completely sequenced genomes. The CD200 orthologs were also identified by conventional RT-PCR followed by Sanger sequencing in the other two CoVs that were unsuccessfully sequenced by NGS. Overall, the CD200 was identified by both sequencing the complete genomes (NGS) and the partial RT-PCR (Sanger sequencing) fragment in eight out of 12 EriCoVs tested. This finding is consistent with a template-switching mechanism deemed to underlie the high rate of RNA recombination in CoVs and consequently to represent a possible mechanism at the origin of recombinant $\mathrm{CoVs}$ including other $\mathrm{CoV}$ or even host related sequences $[27,28]$.

The cellular CD200 protein, with an immune-modulatory effect, is able to suppress, by binding its receptor CD200R, the cascade of inflammatory responses and induce immune tolerance $[29,30]$. The CD200/CD200R interaction is an immunological checkpoint, which in the host prevents the excessive immune-mediated pathology induced as response of the host to infections. Indeed, the inhibitory effect of the CD200/CD200R may impair clearance of the virus. Many viruses have incorporated into their genomes the CD200 orthologs which have been shown to bind the host CD200R and cause downregulation of myeloid activity in the host [31-34] or to interact with the human CD200R diminishing the activation of macrophages and other immune cells [31,35-37]. Inhibition of the CD200-CD200R have a positive effect on coronavirus infections [38], restoring the IFN cascade and clearance of the virus, suggesting that it could also be a possible target for drugs which could reduce the damage caused by inflammatory response caused by the viral infection [39].

In the present study, analyses of the amino acid sequence and the predicted protein secondary structure suggest that the detected Italian EriCoV CD200 orthologs and the hedgehog CD200 could share the same beta strands and most of the sites involved in the binding interface with the mouse CD200R. Differently, one EriCoV strain detected (50265/1) showed a deletion of the beta strand involved in CD200R binding. However, the presence of an additional TSR-B sequence preceding each CD200 orthologs ORF is suggestive of an important role in the regulation of expression during the viral cycle.

We hypothesize that the EriCoV CD200 may bind the host CD200R since the predicted encoded proteins of the CoVs have high amino acid identities and a highly similar secondary structure in the first Ig-like domain necessary for CD200R binding, whose prediction was based on the well characterized mouse CD200 [32]. To confirm this hypothesis in vitro studies are required [40,41]. It would be 
interesting to evaluate if this event, observed in hedgehogs from both urban and rural areas (Figure 2), is limited to EriCoV of this restricted study area or is widespread in hedgehogs' CoV populations.

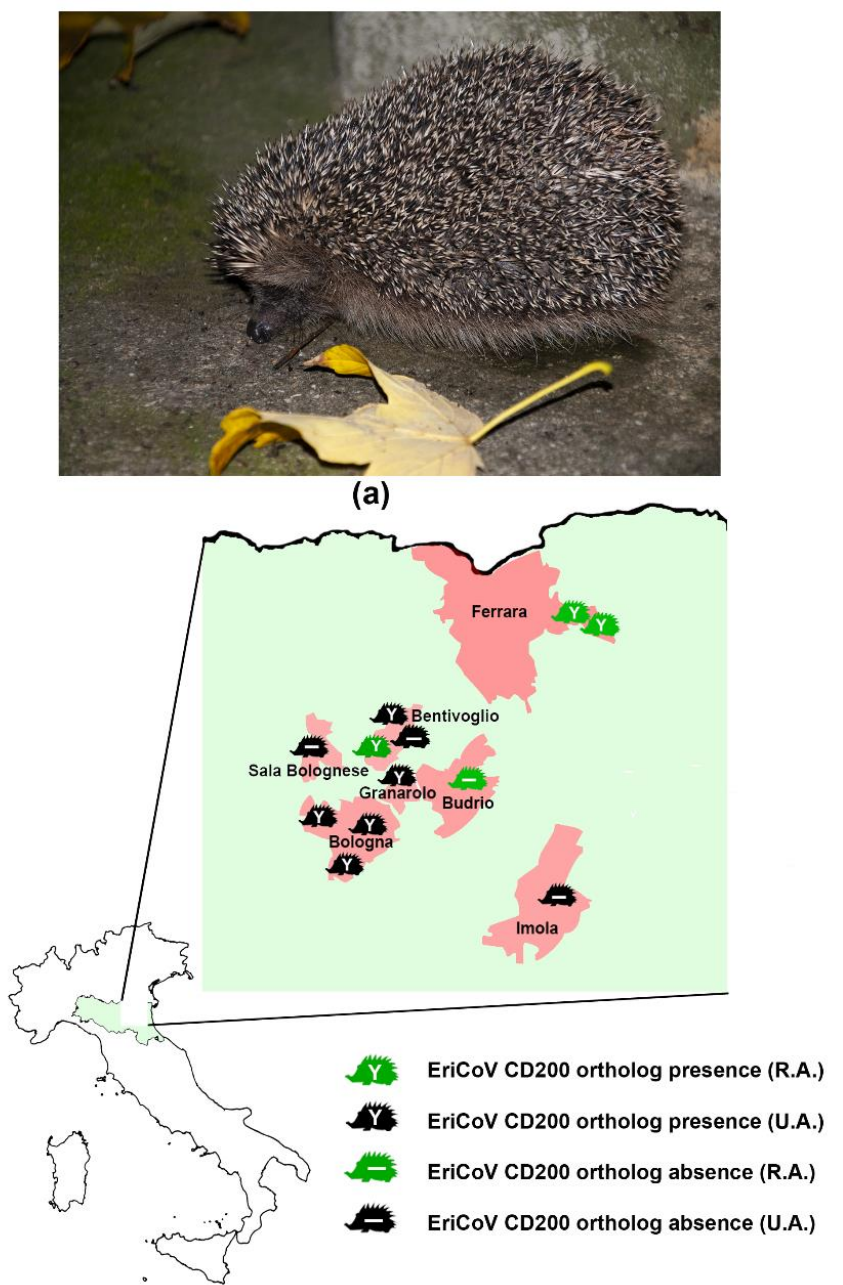

(b)

Figure 2. (a) Nocturnal image of Western European hedgehog (Erinaceus europaeus) from Northern Italy. (b) Viral CD200 ortholog in EriCoV strains from 12 hedgehogs sampled in rural areas (R.A.) and urban areas (U.A.) of Northern Italy. See Table 1 for details.

It is well known that CoVs as other RNA viruses are characterized by high evolutionary rate, due to the lack of proofreading activity of RNA-dependent RNA-polymerase. Although possible recombination events have been reported between bat CoVs and the CoVs from E. amurensis in China, in the present study recombination events between either Italian EriCoVs and MERS-related CoVs from Italian bats (Emilia Romagna region, 2011) [23] or other Merbecovirus strains were excluded.

Phylogenetic analysis conducted on the eight full genomes of the EriCoVs showed their evolutionary correlation with the other strains previously reported in German and Great Britain in Erinaceus europaeus. Differently, the EriCoVs detected are distant from the novel species CoV HKU31 that was only reported in Amur hedgehogs (Erinaceus amurensis) from China. This result, despite the few complete genomes available at the NCBI, may suggest that, as reported in bats, different hedgehogs' species, occupying distant and unconnected areas, may be reservoir of different CoVs species.

By Bayesian analyses, the origin date of the EriCoVs was approximately 1829, confirming previous result [15] obtained with ORF1ab of HKU31 genomes, suggesting that the hedgehog CoVs may have recently emerged and suggesting that an even higher variability could arise in the future. More studies and sequences are required to understand the evolution and diversification of Merbecovirus members. 
As resulted of sequence and phylogenetic analysis of the 8 Italian EriCoV strains obtained from animals captured in seven municipalities, different viral strains circulated in hedgehogs $(95-97 \% \mathrm{nt}$. id.). Only two strains $(50265 / 1,50265 / 13)$ detected in animals captured in two municipalities (Bentivoglio, Granarolo) approximatively $9 \mathrm{~km}$ apart from each other were identical. The heterogeneity of EriCoV strains reported in our study, provide further evidences that hedgehogs are important wild reservoir of MERS-like CoVs. Further studies will be needed to better elucidate the heterogeneity of MERS-CoVs related strains in hedgehogs.

Finally, the possible implications for public and veterinary health related to the circulation of EriCoV in Italian hedgehogs were evaluated. We showed that prediction of the structure of the RBD domain, within the spike protein, of Italian EriCoVs presented a deletion of the loop region between $\beta 7$ and $\beta 8$ which is present in the MERS/HKU4-RDB and necessary for the receptor engagement [42]. The results lead to the hypothesis that hedgehog CoVs may not bind human receptor, however, since some CoVs may use co-receptors as well [43], this may not be a sufficient reason to prevent the virus from crossing the species barrier. Moreover, changing of the protein cannot be ruled out in the future and continuous surveillance is required to monitor the occurrence of mutations or other events, considering that few amino acid changes could determine the adaptation to novel hosts including humans. According to results previously obtained from a limited hedgehogs' number, a sustained EriCoV circulation seems occur in the study area. Indeed, $58.3 \%$ of fecal samples collected in the Emilia-Romagna region of Northern Italy from 24 hedgehogs tested PCR-positive for EriCoVs, showing no clinical disease related to this infection [16]. These findings further support the hypothesis that hedgehogs could be considered natural reservoirs of CoVs and also act as chronic shedding carriers of these potentially emerging RNA viruses. In this context, particular attention must be paid to the management of hedgehogs admitted to multi-species wildlife rehabilitation centers [44], taking into account the high mutation rates characterizing members of the Coronaviridae family and their possible successful interspecies host jumps [45]. In particular, since hedgehogs and bats are primarily nocturnal and insectivorous mammals [20-22,46] playing the role of Merbecovirus reservoir, both direct and indirect contacts between them should be avoided to prevent possible cross species infection and recombination events. Finally, to avoid the man-made spread of EriCoVs into the wild, the return of rehabilitated hedgehogs to their original recovery areas is recommended.

\section{Conclusions}

We describe for the first time the acquisition of the host gene CD200 by Beta-CoVs. This event has been observed in a wild hedgehog population naturally infected with CoVs, sampled in Italy. The obtained result is important since evidenced the chance that CoVs can acquire and eventually use the host protein to their advantage. The incorporation of a gene involved with the immune-modulatory cascade could allow the virus to escape the host defence.

The acquisition by CoVs of a glycoprotein with multiple function types in the host needs further research, as it might affect the immunology, pathophysiology, and epidemiology of these potentially emerging pathogens. Further studies are required to better investigate the role played by these insectivorous mammals as $\mathrm{CoVs}^{\prime}$ reservoir and to delineate the animal origin of Merbecovirus.

Finally, taking into account the high mutation rates characterizing members of the Coronaviridae family and their possible successful interspecies host jumps, we have recommended precautionary health measures to be taken into account during the hedgehogs' management at multi-species wildlife rehabilitation centers until their release into the wild.

Supplementary Materials: The following are available online at http://www.mdpi.com/1999-4915/12/12/1471/s1, Table S1: Genome localization of Italian EriCoVs predicted protein sequences, length and putative leader TRS, Table S2: Prediction of the putative pp1a/pp1b cleavage sites of Italian EriCoVs based on sequence comparison with Erinaceus/VMC/DEU/2012 (NC_039207.1), Table S3: Amino acids identities (\%) between predicted proteins of the Italian EriCoVs and prototype clade c Betacoronaviruses, Table S4: Amino acids identities (\%) between predicted CD200 ortholog from Italian EriCoVs and human, mouse and hedgehog CD200, Figure S1: Maximum Likelihood phylogenetic tree of CD200 sequences reported in mammals and viral orthologs, Figure S2: Alignment 
of amino acid sequences and secondary structure of CD200 orthologs found in Italian EriCoVs with the predicted beta strands (A, B, C, C', C', D, E, F, G), Figure S3: Estimation of tMRCA of members of Merbecovirus using the complete genome sequences of Italian EriCoVs.

Author Contributions: Conceptualization, M.A.D.M., M.D., G.V., and A.M.; methodology, L.D.S. and I.D.B.; software, L.D.S.; formal analysis, L.D.S.; investigation, D.L. and C.C.; data curation, L.D.S.; writing-original draft preparation, L.D.S., I.D.B., and G.V.; writing-review and editing L.D.S., I.D.B., G.V., D.L., M.A.D.M., C.C., and A.M.; supervision G.V., A.M., M.D., and M.A.D.M. All authors have read and agreed to the published version of the manuscript.

Funding: This research received no external funding.

Acknowledgments: The authors thank Adriano De Faveri (ISPRA) for the valuable assistance in figure preparation and for providing the image used. We also thank all the staff of the "Otus" wildlife treatment and rehabilitation center (LIPU-Bologna), in particular Nadia Caselli for their kind assistance during sample collection. We wish to thank Marco Crescenzi, Manuela Marra and Maria Carollo from the ISS Core Facilities Technical-Scientific Service for the Next Generation Sequencing through Ion GeneStudio S5 System.

Conflicts of Interest: The authors declare no conflict of interest.

\section{References}

1. Wang, L.; Zhang, Y. Animal coronaviruses: A brief introduction. In Animal Coronaviruses; Springer Protocols Handbooks; Wang, L., Ed.; Humana Press: New York, NY, USA, 2016; pp. 3-11.

2. Greenberg, S.B. Update on human rhinovirus and coronavirus infections. Semin. Respir. Crit. Care Med. 2016, 37, 555-571. [CrossRef] [PubMed]

3. Wong, A.C.P.; Li, X.; Lau, S.K.P.; Woo, P.C.Y. Global epidemiology of bat coronaviruses. Viruses 2019, 11, 174. [CrossRef] [PubMed]

4. World Health Organization. Disease Outbreak News Update: Middle East Respiratory Syndrome Coronavirus (MERS-CoV). Available online: https://www.who.int/csr/don/02-jul-2020-mers-saudi-arabia/en/ (accessed on 16 November 2020).

5. Cui, J.; Li, F.; Shi, Z.L. Origin and evolution of pathogenic coronaviruses. Nat. Rev. Microbiol. 2019, 17, 181-192. [CrossRef]

6. Raj, V.S.; Mou, H.; Smits, S.L.; Dekkers, D.H.; Muller, M.A.; Dijkman, R.; Muth, D.; Demmers, J.A.; Zaki, A.; Fouchier, R.A.; et al. Dipeptidyl peptidase 4 is a functional receptor for the emerging human coronavirus-EMC. Nature 2013, 495, 251-254. [CrossRef] [PubMed]

7. Ge, X.Y.; Hu, B.; Shi, Z.L. Bat Coronaviruses. In Bats and Viruses: A New Frontier of Emerging Infectious Diseases; Wang, L.F., Cowled, C., Eds.; John Wiley Sons Inc.: Hoboken, NJ, USA, 2015.

8. Beltz, L.A. Bats and Human Health: Ebola, SARS, Rabies and Beyond; John Wiley \& Sons Inc.: Hoboken, NJ, USA, 2018.

9. Lau, S.K.P.; Zhang, L.; Luk, H.K.H.; Xiong, L.; Peng, X.; Li, K.S.M.; He, X.; Zhao, P.S.; Fan, R.Y.Y.; Wong, A.C.P.; et al. Receptor usage of a novel bat lineage $\mathrm{c}$ betacoronavirus reveals evolution of Middle East respiratory syndrome-related coronavirus spike proteins for human dipeptidyl peptidase 4 Binding. J. Infect. Dis. 2018, 218, 197-207. [CrossRef]

10. Zhou, P.; Yang, X.L.; Wang, X.G.; Hu, B.; Zhang, L.; Zhang, W.; Si, H.R.; Zhu, Y.; Li, B.; Huang, C.L.; et al. A pneumonia outbreak associated with a new coronavirus of probable bat origin. Nature 2020, 579, 270-273. [CrossRef]

11. Onuma, M.; Cao, Y.; Hasegawa, M.; Kusakabe, S. A Close relationship of Chiroptera with Eulipotyphla (Core Insectivora) suggested by four mitochondrial genes. Zool. Sci. 2000, 17, 1327-1332. [CrossRef]

12. Corman, V.M.; Kallies, R.; Philipps, H.; Göpner, G.; Müller, M.A.; Eckerle, I.; Brünink, S.; Drosten, C.; Drexler, J.F. Characterization of a novel betacoronavirus related to middle East respiratory syndrome coronavirus in European hedgehogs. J. Virol. 2014, 88, 717-724. [CrossRef]

13. Monchatre-Leroy, E.; Boué, F.; Boucher, J.M.; Renault, C.; Moutou, F.; Ar Gouilh, M.; Umhang, G. Identification of alpha and beta coronavirus in wildlife species in France: Bats, rodents, rabbits, and hedgehogs. Viruses 2017, 9, 364. [CrossRef]

14. Saldanha, I.F.; Lawson, B.; Goharriz, H.; Fernandez, J.R.-R.; John, S.K.; Fooks, A.R.; Cunningham, A.A.; Johnson, N.; Horton, D.L. Extension of the known distribution of a novel clade $C$ betacoronavirus in a wildlife host. Epidemiol. Infect. 2019, 147, e169. [CrossRef] 
15. Lau, S.; Luk, H.; Wong, A.; Fan, R.; Lam, C.; Li, K.; Ahmed, S.S.; Chow, F.; Cai, J.P.; Zhu, X.; et al. Identification of a novel Betacoronavirus (Merbecovirus) in amur hedgehogs from China. Viruses 2019, 11, 980. [CrossRef] [PubMed]

16. Delogu, M.; Cotti, C.; Lelli, D.; Sozzi, E.; Trogu, T.; Lavazza, A.; Garuti, G.; Castrucci, M.R.; Vaccari, G.; De Marco, M.A.; et al. Eco-virological preliminary study of potentially emerging pathogens in hedgehogs (Erinaceus europaeus) recovered at a wildlife treatment and rehabilitation center in northern Italy. Animals 2020, 10, 407. [CrossRef] [PubMed]

17. Ziebuhr, J.; Baric, R.S.; Baker, S.; de Groot, R.J.; Drosten, C.; Gulyaeva, A.; Haagmans, B.L.; Lauber, C.; Neuman, B.W.; Perlman, S.; et al. Create $12 \mathrm{New}$ Species in the Family Coronaviridae. Taxonomic Proposal: 2015.003a-eS International Committee on Taxonomy of Viruses (ICTV). Available online: https: //talk.ictvonline.org/ICTV/proposals/2015.003a-eS.A.v2.Coronaviridae_12sp.pdf (accessed on 18 November 2020).

18. Douady, C.J.; Douzery, E.J.P. Hedgehogs, shrews, moles, and solenodons (Eulipotyphla). In The Timetree of Life; Hedges, S.B., Kumar, S., Eds.; Oxford University Press: New York, NY, USA, 2009; pp. 495-498.

19. Tsagkogeorga, G.; Parker, J.; Stupka, E.; Cotton, J.A.; Rossiter, S.J. Phylogenomic analyses elucidate the evolutionary relationships of bats. Curr. Biol. 2013, 23, 2262-2267. [CrossRef] [PubMed]

20. Rondinini, C.; Capasso, S. Erinaceus europaeus Italian IUCN Committee, Ministry of the Environment and Protection of the Territory and the Sea, Federation of Parks [Comitato Italiano IUCN, Ministero dell'Ambiente e della Tutela del Territorio e del Mare, Federparchi] 2013. Available online: http://www.iucn.it/scheda.php? id=-944215748 (accessed on 16 November 2020).

21. Rondinini, C.; Capasso, S. Erinaceus roumanicus Italian IUCN Committee, Ministry of the Environment and Protection of the Territory and the Sea, Federation of Parks [Comitato Italiano IUCN, Ministero dell'Ambiente e della Tutela del Territorio e del Mare, Federparchi] 2013. Available online: http://www.iucn.it/scheda.php? $\mathrm{id}=1308809394$ (accessed on 16 November 2020).

22. G.I.R.C. Gruppo Italiano Ricerca Chirotteri, Specie Associazione Teriologica Italiana, [G-I.R.C. Italian Chiroptera Research Group, Species, Italian Mammal Society]. Available online: https://www.mammiferi. org/girc/specie/ (accessed on 16 November 2020).

23. Moreno, A.; Lelli, D.; de Sabato, L.; Zaccaria, G.; Boni, A.; Sozzi, E.; Prosperi, A.; Lavazza, A.; Cella, E.; Castrucci, M.R.; et al. Detection and full genome characterization of two beta CoV viruses related to Middle East respiratory syndrome from bats in Italy. Virol. J. 2018, 15, 10. [CrossRef] [PubMed]

24. Djikeng, A.; Halpin, R.; Kuzmickas, R.; Depasse, J.; Feldblyum, J.; Sengamalay, N.; Afonso, C.; Zhang, X.; Anderson, N.G.; Ghedin, E.; et al. Viral genome sequencing by random priming methods. BMC Genom. 2008, 9, 5. [CrossRef] [PubMed]

25. Pettersen, E.F.; Goddard, T.D.; Huang, C.C.; Couch, G.S.; Greenblatt, D.M.; Meng, E.C.; Ferrin, T.E. UCSF Chimera-A visualization system for exploratory research and analysis. J. Comput. Chem. 2004, 25, 1605-1612. [CrossRef] [PubMed]

26. Robert, X.; Gouet, P. Deciphering key features in protein structures with the newENDscript server. Nucl. Acids Res. 2014, 42, W320-W324. [CrossRef]

27. Makino, S.; Keck, J.G.; Stohlman, S.A.; Lai, M.M. High-frequency RNA recombination of murine coronaviruses. J. Virol. 1986, 57, 729-737. [CrossRef]

28. Seyran, M.; Pizzol, D.; Adadi, P.; El-Aziz, T.M.A.; Hassan, S.S.; Soares, A.; Kandimalla, R.; Lundstrom, K.; Tambuwala, M.; Aljabali, A.A.A.; et al. Questions concerning the proximal origin of SARS-CoV-2. J. Med. Virol. 2020. [CrossRef]

29. Vaine, C.A.; Soberman, R.J. The CD200-CD200R1 inhibitory signaling pathway: Immune regulation and host-pathogen interactions. Adv. Immunol. 2014, 121, 191-211.

30. Mousavinezhad-Moghaddam, M.; Amin, A.A.; Rafatpanah, H.; Rezaee, S.A. A new insight into viral proteins as Immunomodulatory therapeutic agents: KSHV vOX2 a homolog of human CD200 as a potent anti-inflammatory protein. Iran. J. Basic Med. Sci. 2016, 19, 2-13. [PubMed]

31. Foster-Cuevas, M.; Wright, G.J.; Puklavec, M.J.; Brown, M.H.; Barclay, A.N. Human herpesvirus 8 K14 protein mimics CD200 in down- regulating macrophage activation through CD200 receptor. J. Virol. 2004, 78, 7667-7676. [CrossRef] [PubMed] 
32. Rosenblum, M.D.; Olasz, E.B.; Yancey, K.B.; Woodliff, J.E.; Lazarova, Z.; Gerber, K.A.; Truitt, R.L. Expression of CD200 on epithelial cells of the murine hair follicle: A role in tissue-specific immune tolerance? J. Investig. Dermatol. 2004, 123, 880-887. [CrossRef] [PubMed]

33. Zhang, L.; Stanford, M.; Liu, J.; Barrett, C.; Jiang, L.; Barclay, A.N.; McFadden, G. Inhibition of macrophage activation by the myxoma virus M141 protein (vCD200). J. Virol. 2009, 83, 9602-9607. [CrossRef]

34. Foster-Cuevas, M.; Westerholt, T.; Ahmed, M.; Brown, M.H.; Barclay, A.N.; Voigt, S. Cytomegalovirus e127 protein interacts with the inhibitory CD200 receptor. J. Virol. 2011, 85, 6055-6059. [CrossRef]

35. Rezaee, S.A.; Gracie, J.A.; McInnes, I.B.; Blackbourn, D.J. Inhibition of neutrophil function by the Kaposi's sarcoma-associated herpesvirus vOX2 protein. AIDS 2005, 19, 1907-1910. [CrossRef]

36. Shiratori, I.; Yamaguchi, M.; Suzukawa, M.; Yamamoto, K.; Lanier, L.L.; Saito, T.; Arase, H. Down-regulation of basophil function by human CD200 and human herpesvirus-8 CD200. J. Immunol. 2005, 175, 4441-4449. [CrossRef]

37. Misstear, K.; Chanas, S.A.; Rezaee, S.A.; Colman, R.; Quinn, L.L.; Long, H.M.; Goodyear, O.; Lord, J.M.; Hislop, A.D.; Blackbourn, D.J. Suppression of antigen-specific T cell responses by the Kaposi's sarcoma-associated herpesvirus viral OX2 protein and its cellular orthologue, CD200. J. Virol. 2012, 86, 6246-6257. [CrossRef]

38. Karnam, G.; Rygiel, T.P.; Raaben, M.; Grinwis, G.C.M.; Coenjaerts, F.E.; Ressing, M.E.; Rottier, P.J.M.; de Haan, C.A.M.; Meyaard, M. CD200 receptor controls sex-specific TLR7 responses to viral infection. PLoS Pathog. 2012, 8, e1002710. [CrossRef]

39. Ceribelli, A.; Motta, F.; De Santis, M.; Ansari, A.A.; Ridgway, W.M.; Gershwin, M.E.; Selmi, C. Recommendations for coronavirus infection in rheumatic diseases treated with biologic therapy. J. Autoimmun. 2020, 109, 102442. [CrossRef]

40. Cameron, C.M.; Barrett, J.W.; Liu, L.; Lucas, A.R.; McFadden, G. Myxoma virus M141R expresses a viral CD200 (vOX-2) that is responsible for down-regulation of macrophage and T-cell activation in vivo. J. Virol. 2005, 79, 6052-6067. [CrossRef] [PubMed]

41. Akkaya, M.; Kwong, L.S.; Akkaya, E.; Hatherley, D.; Barclay, A.N. Rabbit CD200R binds host CD200 but not CD200-like proteins from poxviruses. Virology 2016, 488, 1-8. [CrossRef] [PubMed]

42. Wang, Q.; Qi, J.; Yuan, Y.; Xuan, Y.; Han, P.; Wan, Y.; Ji, W.; Li, Y.; Wu, Y.; Wang, J.; et al. Bat origins of MERS-CoV supported by bat coronavirus HKU4 usage of human receptor CD26. Cell Host Microbe 2014, 16, 328-337. [CrossRef] [PubMed]

43. Solerte, S.B.; Di Sabatino, A.; Galli, M.; Fiorina, P. Dipeptidyl peptidase-4 (DPP4) inhibition in COVID-19. Acta Diabetol. 2020, 57, 779-783. [CrossRef] [PubMed]

44. Riley, P.Y.; Chomel, B.B. Hedgehog zoonoses. Emerg. Infect. Dis. 2005, 11, 1-5. [CrossRef] [PubMed]

45. Su, S.; Wong, G.; Shi, W.; Liu, J.; Lai, A.C.K.; Zhou, J.; Liu, W.; Bi, Y.; Gao, G.F. Epidemiology, genetic recombination, and pathogenesis of coronaviruses. Trends Microbiol. 2016, 24, 490-502. [CrossRef]

46. Morris, P.A. A study of home range and movements in the hedgehog (Erinaceus europaeus). J. Zool. Lond. 1988, 214, 433-499. [CrossRef]

Publisher's Note: MDPI stays neutral with regard to jurisdictional claims in published maps and institutional affiliations.

(C) 2020 by the authors. Licensee MDPI, Basel, Switzerland. This article is an open access article distributed under the terms and conditions of the Creative Commons Attribution (CC BY) license (http://creativecommons.org/licenses/by/4.0/). 